\title{
MicroRNA: biogenesis and computational target identification: a review
}

\author{
Amod Kumar ${ }^{1}$, V. N. Muhasin Asaf ${ }^{1}$, Kush Srivastava ${ }^{1}$, Abdul Rahim ${ }^{1}$, J. K. Chaudhary ${ }^{2}$ and Manjit Panigrahi ${ }^{3}$ \\ 1. Division of Animal Genetics, Indian Veterinary Research Institute, Izatnagar - 243122, Dist. Bareilly, Uttar Pradesh, \\ India; 2. Division of Livestock Economics, Statistics and Information Technology, Indian Veterinary Research Institute, \\ Izatnagar - 243122, Dist. Bareilly, Uttar Pradesh, India; 3. Scientist, Division of Animal Genetics, Indian Veterinary \\ Research Institute, Izatnagar - 243122, Dist. Bareilly, Uttar Pradesh, India \\ Corresponding author: Amod Kumar, email: amodvet@gmail.com \\ Received: 10-06-2013, Revised: 07-07-2013, Accepted: 09-07-2013, Published online: 16-08-2013
}

doi: 10.14202/vetworld.2013.761-765 How to cite this article: Kumar A, Muhasin Asaf VN, Srivastava K, Abdul Rahim, Chaudhary JK and Panigrahi M (2013) MicroRNA: biogenesis and computational target identification: a review, Veterinary World 6(10): 761-765.

\begin{abstract}
MicroRNAs are a class of small, endogenously produced, 18 to 24 nucleotides long in length. These are non-coding RNAs that regulate the gene expression at post-transcriptional level. They play important roles in animals and plants by controlling regulatory mechanisms, and likely influencing the output of many protein-coding genes. They generally bind to 3' UTR region of the target sequence which then leads to alterations in the gene expression. They also bind to other regions like coding sequence and 5' UTR but these are less efficient sites of interaction compared to 3'UTR. This alteration in gene expression is either due to repression of translation or mRNA degradation whereby the RNA interference pathway is initiated to eliminate the targeted sequences. Now a days, various computational or bioinformatics databases, tools, and algorithms have been developed to identify the target genes which will be further biologically validated using various techniques like reporter gene assay, qRT-PCR, microarray etc.
\end{abstract}

Keywords: miRNA, gene regulation, UTR, bioinformatics

\section{Introduction}

MicroRNAs (miRNA) are endogenously produced, small noncoding RNAs, play important roles in gene expression, and have multiple target binding sites in the mRNAs resulting in their silencing [1]. miRNA expression profiling is now popularized as they are key regulators in gene expression networks, have impact on many biological processes and are now proved to be biomarkers for disease [2]. miRNA change the gene expression by attaching to complementary base pairs in the mRNAs which then lead to translational repression or transcript degradation [3]. Translational repression may be the primary mode of action in animals because of an imperfect duplex formation between miRNA and mRNA, whereas in plants they have perfect binding sites lead to degradation of mRNA [4]. Despite the actual underlying mechanism being still poorly understood, miRNAs seem to be major factors for regulation of different pathways or processes like development, differentiation, proliferation and cell death [4].

miRNAs also emerged as important regulators of metabolism [5]. At the 5' end of the miRNA, a 7-8 nucleotide long region is the primary component of miRNA target specificity which is termed as the seed region [6]. There are two types of miRNA i.e. intronic and exonic, the difference in both being only of polymerases and RNA splicing components required during their biogenesis [7]. miRNAs show phenomena of multiplicity or redundancy and cooperativity.

Copyright: The authors. This article is an open access article licensed under the terms of the Creative Commons Attribution License (http://creativecommons.org/licenses/by/2.0) which permits unrestricted use, distribution and reproduction in any medium, provided the work is properly cited.
Multiplicity indicates that a single miRNA has many target sites, whereas cooperativity denotes that many miRNAs have common target sites [8]. Data generated by new generation sequencing technologies along with miRNA biology enhances our understanding of evolution of miRNA and their targets [9].

\section{Biogenesis of miRNA}

They were originally discovered in Caenorhabditis elegans as post transcriptional generegulators $[4,10]$. In C. elegans, the translation of lin-14 mRNA is regulated by a small non-coding RNA which has many target binding sites in 3'UTR of lin-4 gene [11]. All eukaryotic and several viruses express miRNAs $[4,12]$ but no RNA virus except retrovirus has been found to produce miRNA which was confirmed by computational and experimental analysis on the genome of hepatitis $\mathrm{C}$ virus and yellow-fever virus [13]. They regulate a variety of biological processes like cell growth, tissue differentiation, apoptosis, viral infection and some reports also show their role in the inflammatory process $[4,14]$.

They bind to the 3'UTR region of transcript and inhibit translation or degrade mRNA [15]. They also bind to other regions like coding sequence and 5' UTR but these are less efficient sites of interaction when compared to 3'UTR. A single miRNA can regulate the expression of many genes and modulate many biological pathways [16]. In animals, miRNAs were thought to reduce translation of their targets with very less or no influence on mRNA quantity, whereas opposite effects were thought to be true in plants [17]. Some miRNA genes show very similar expression profile which are clustered and separated by only a few 
nucleotides, suggesting that they are monitored by common promoters [18].

The biogenesis of miRNA is a well conserved process (Figure-1). The microRNA producing gene is first transcribed into primary miRNA, which is further processed by a nuclear RNase type III enzyme, Drosha, into precursor miRNA [19]. The pre-miRNA is then transported to cytoplasm by the nuclear export factor Exportin 5 and a cofactor Ran-GTP. In cytoplasm, the pre-miRNA is again cleaved by Dicer into miRNA: miRNA(In heteroduplex miRNA is less stable) duplex [19]. From miRNA: miRNA (In heteroduplex miRNA is less stable) duplex, one strand is degraded and other is identified by the RNA-induced silencing complex (RISC) [20] which targets the mRNA transcript.

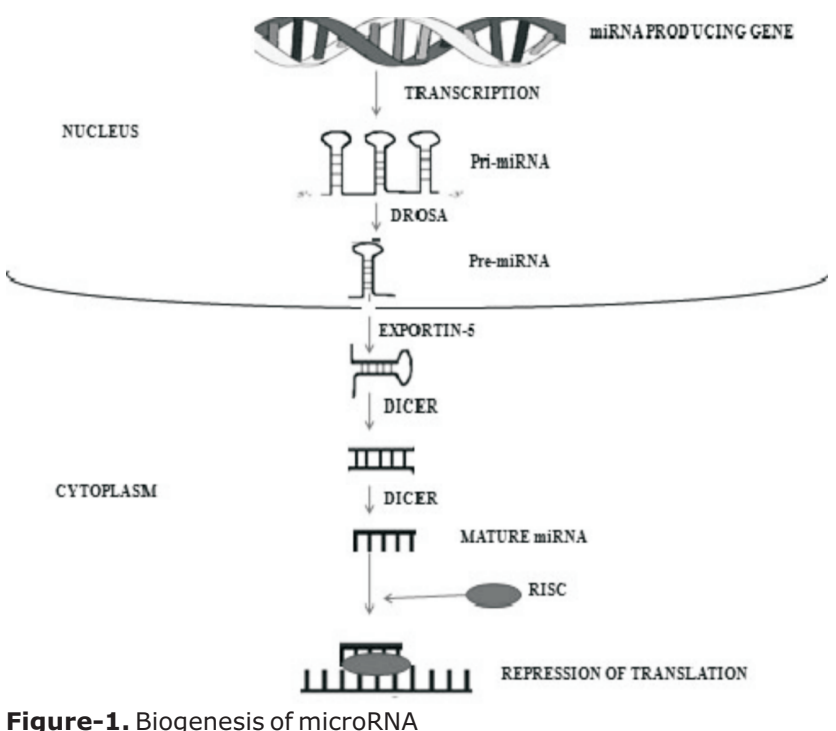

miRNA nomenclature

The miRNA has 3 or 4 prefix letter for a particular species like 'gga' for chicken. The processed final miRNA or mature sequences are nominated as 'miR' in the database, whereas the precursor hairpins for mature miRNAs are labeled as 'mir' [21]. Orthologous miRNAs differ only in the first letter prefixes like hsamiR-101 in human and mmu-miR-101 in mouse. However, in case of paralogous sequences whose mature microRNAs vary at only one or two positions are given lettered suffixes like gga-miR-10a and ggamiR-10b in chicken genome.

\section{Regulation of translation by miRNA}

It is confirmed by many researchers that miRNA reduces translation of protein-coding genes [22]. Some evidences have also emerged that contradicts the current dogma of decreased gene translation [23]. These regulatory mechanisms include mRNA degradation by removing the cap at 5 ' end and deadenylation of the poly-A tail of mRNA result in repression of translation $[24,25]$. Another study dealing with the miRNA mechanism of translational repression revealed that mRNA abundance did not always decrease with gene translation, indicating that it may involve a different mechanism [26]. However, instead of mRNA degradation, it may start a cascade for blocking initiation of proteins from binding to the $5^{\prime}$ cap of the mRNA [27]. An additional mechanism of translational repression is by translocation of the miRNA:mRNA complex to cytoplasmic foci in the cell, known as processing bodies (P-bodies), after the miRNA:RISC complex binds to the mRNA target [28].

\section{Principles of miRNA targets}

The miRNA target prediction principles used by most of the approaches are almost similar [29] such as seed sequence complementarity [30], thermodynamic stability, conserved sites etc.

1. Seed sequence complementarity: At the 5 'end of the miRNA, a 2-7 nucleotide region called as 'seed sequence' binds within mRNA target site. This WatsonCrick paring of miRNA and mRNA is important factor of target gene prediction [31]. Along with this seed pairing, there is pairing at $3^{\prime}$ end of miRNA from 13-16 nucleotide (3-4 nucleotides) called as '3'-supplementary' pairing which leads to increased specificity and affinity. Furthermore for compensating a mismatch in seed region, Watson-Crick paring of miRNA and mRNA at 3' end of miRNA is called as '3'-compensatory sites' [31].

2. Conservation: Identification of a conserved site as miRNA target significantly decreases the falsepositive rates of prediction programs. Sites are commonly regarded as conserved if they are retained at orthologous locations in multiple genomes, which mean they have to appear exactly at the same position in the alignment of the 3'UTR sequences [32].

3. Thermodynamics: Thermodynamic stability is another filter criteria for target gene prediction. The more negative free energy of miRNA:target duplex, the higher energy is required to disrupt this duplex formation. Thus, a RNA duplex is in a thermodynamically more stable state.

4. Accessibility of target site: The accessibility of the target sites were evaluated based on the secondary structure of the target mRNA. The secondary structure of the mRNA appears also to play an important role. For binding to the miRNA the target site has to be accessible, which means it has to be opened and must not interact with other sites within the mRNA [33, 34]. Two types of folds, namely the 'global' fold which was the secondary structure of the complete coding sequence and the 'regional' fold which was the secondary structure obtained by folding about 220 bases with 100 bases on either sides of the target site sequence can be used for target site accessibility. The MFE of regional folding with constraint of unpairing at the target site ( $\left.\Delta \mathrm{G}_{\text {unpaired }}\right)$ compared with that of the native fold $\left(\Delta \mathrm{G}_{\text {free }}\right)$ to assess the free energy of unfolding $\left(\Delta \mathrm{G}_{\text {open }}\right)$.

The accessibility energy of the miRNA target sites were calculated as:

Accessibility energy $(\Delta \Delta \mathrm{G})=\Delta \mathrm{G}_{\text {duplex }}-\Delta \mathrm{G}_{\text {open }},($ where 
Table-1. Features of miRNA target prediction algorithms [44].

\begin{tabular}{|c|c|c|c|c|}
\hline \multirow[t]{2}{*}{ Name of the program } & \multicolumn{3}{|c|}{ Features of different algorithms } & \multirow{2}{*}{$\begin{array}{l}\text { Approach } \\
\text { rule based }\end{array}$} \\
\hline & Seed match & Free energy & Conservation & \\
\hline TargetScan & Yes & Yes & Yes & Rule based \\
\hline TargetScanS & Yes & - & Yes & Rule based \\
\hline miRanda & - & Yes & Yes & Rule based \\
\hline Pita & Yes & Yes & --- & Rule based \\
\hline DIANA-microT & Yes & Yes & --- & Rule based \\
\hline RNAhybrid & Yes & Yes & --- & Rule based \\
\hline microlnspector & Yes & Yes & --- & Rule based \\
\hline MovingTargets & Yes & Yes & --- & Rule based \\
\hline Nucleus & Yes & Yes & --- & Rule based \\
\hline Pictar & Yes & Yes & Yes & Data Driven: HMM \\
\hline miTarget & Yes & Yes & & Data Driven: SVM \\
\hline mirTarget & Yes & Yes & Yes & Data Driven: SVM \\
\hline rna22 & Yes & Yes & & Data Driven: Markov Chain \\
\hline SVMicro & Yes & Yes & Yes & Data Driven: SVM \\
\hline Targetboost & - & - & --- & Data Driven: Boost \\
\hline GenMiR++ & Yes & Yes & Yes & Data Driven: Bayesian Learning \\
\hline
\end{tabular}

$\left.\Delta \mathrm{G}_{\text {open }}=\Delta \mathrm{G}_{\text {free }}-\Delta \mathrm{G}_{\text {unpaired }}\right)$

\section{Types of miRNA target sites}

miRNAs down-regulate gene expression mostly by imperfect binding to complementary sites within mRNA which lead to reduction of translation, mRNA degradation or induce their cleavage. There are three types of miRNA target sites. This includes Canonical, Marginal, and Atypical target sites [31].

Canonical target sites: It has a complete pairing within the seed region which determines the certainty of the interaction and account for the majority of validated conserved targets [35]. These sites include 7mer-A1 site, $7 \mathrm{mer}-\mathrm{m} 8$ site and $8 \mathrm{mer}$ site. The $7 \mathrm{mer}-\mathrm{A} 1$ site has 2-7 seed match plus nucleotide $A$ at 1 position in mRNA, 7mer-m8 site has 2-7 seed match plus a match at position 8 in mRNA and 8 mer site has combination of above two sites [31].

Marginal sites: It includes 6 nucleotides Watson-Crick pairing in the seed region. These are 6 mer site and offset 6 mer sites. The 6 mer sites have matching at 2-7 seed region, where an offset 6 mer site has matching at 3-8 positions. These $6 \mathrm{mer}$ sites typically have reduced efficacy and are conserved by chance more frequently than the larger sites. In stringent seed pairing criteria for site efficacy and prediction efficiency, 6mer sites are ignored [31].

Atypical sites: It includes 3'-supplementary and 3'compensatory sites. For 3'-supplementary sites, WatsonCrick pairing usually centering miRNA nucleotides 13-16 supplements the seed matching which increase the efficacy [36]. For 3'-compensatory sites, Watson -Crick pairing usually centering on miRNA nucleotides 13-16 can compensate for a seed mismatch and thereby create a functional site. Now, it is known that simple base pairing is not sufficient for miRNA target prediction [37] and the secondary structure of miRNAmRNA duplexes is a factor that should be taken into consideration [38].

Many existing algorithms based on conservation analyses, also include the binding energy of miRNA-
mRNA duplex calculations into a final score.

\section{Algorithms for miRNA target prediction}

Computational target prediction algorithm which is a rule-based algorithm came to existence since TargetScan [39] was proposed in 2003 and is still among the most popular algorithms. It is based on simple discriminative rules derived from important features of target recognition observed from experiments. The rule-based algorithms include TargetScan [39], miRanda [40], PITA [33], etc. In recent years, new data-driven prediction algorithms emerge along with the improving knowledge of miRNA target recognition and the increasing availability of various types of relevant data sets. Data driven algorithms rely on important discriminative features learned from data using sophisticated models. It includes MirTarget [41], PicTar [42], miTarget [43], etc. Features of different target prediction algorithms are depicted in Table-1 while some useful databases related to miRNA target prediction are summarized in Table-2.

Validation of miRNA target genes: Because of insufficient efficacy and specificity of different target prediction tools, there is a need to biological validation of predicted target genes. The reporter gene assay is used most commonly for assessing miRNA:mRNA interactions [45]. In this method, the 3' UTR of the mRNA is cloned downstream of a luciferase ORF. When this vector is transfected into cells expressing the targeting miRNA, luciferase activity should be lower for the empty vector. Microarray analysis can be used to observe miRNA:mRNA interactions [46] which measure changes of mRNA levels. MicroRNA profiling data obtained from sequencing of small RNAs can also be validated by qRT-PCR. Another approach to verify a miRNA-target interaction would be to knockout the miRNA gene and examine the effects on protein changes [31]. Northern blotting can be useful, although time-consuming, especially in those cases where different isoforms of the target are expressed. The effect of miRNA on target gene can also be tested at protein level by western blotting, ELISA etc. 
Table-2. Online resources for miRNA target prediction [44]

\begin{tabular}{ll}
\hline Category & Website \\
\hline Genome of different species & NCBI FTP(ftp://ftp.ncbi.nih.gov/genomes/) UCSC \\
Homologous gene information & UCSC (http://genome.ucsc.edu/) \\
Sequence and information of miRNAs & miRBase(http://microrna.sanger.ac.uk/sequences/index.shtml) \\
Experimentally validated miRNA targets & TarBase(http://diana.cslab.ece.ntua.gr/tarbase/) \\
Computational predicted targets & miRecords(http://miRecords.umn.edu/miRecords) \\
\hline
\end{tabular}

Some miRNA target prediction algorithms: By using computational or bioinformatics tools one can predict the targets and then go for validation by experimentation. Some of the miRNAs target prediction algorithms are discussed below:

TargetScan and TargetScans: TargetScan predicts biological targets of miRNAs by searching for the presence of conserved $8 \mathrm{mer}$ and 7 mer sites that match the seed region of each miRNA and optional nonconserved sites are also predicted. It also identifies sites with mismatches in the seed region that are compensated by conserved 3 ' end [15], and then check the complementarity in other regions. It also uses conservation criteria for filtering and Vienna RNA folding package for thermodynamic evaluation. The estimated false-positive rate is between $22 \%$ and $31 \%$. TargetScanS is the improved version of TargetScan.

miRDB: It is an online database system for miRNA target gene prediction in five species: human, mouse, rat, dog and chicken. miRDB is different from existing miRNA functional databases mainly in the following: (i) Originality of the target prediction results; (ii) a new database design strategy centered on mature miRNAs; and (iii) a wiki editing interface for communityprovided miRNA annotations [41]. Compared to other databases, miRNA functional annotations in miRDB are presented with a major focus on mature miRNAs.

miRecords: It is an integrated resource for animal miRNA-target interactions. It is a database of two components i.e. experimentally validated and computationally predicted miRNA targets [32]. The validated targets component of the miRecords contains validated miRNA-target interactions with systematic documentation of experimental support for such interactions. The Predicted Targets component of miRecords is an integration of predicted miRNA targets produced by 11 established miRNA target prediction programs. miRecords is expected to serve as a useful resource not only for experimental miRNA researchers, but also for informatics scientists developing the next-generation miRNA target prediction programs.

miRanda: It finds potential binding sites by searching for high complementarity between miRNA and mRNA [40]. The algorithm favors complementarity between $5^{\prime}$ end of the miRNA and 3' end of mRNA and then evaluate thermodynamically, using the Vienna RNA folding package. mirSVR, a regression model that is trained on sequence and contextual features of the predicted miRNA:mRNA duplex is used for target site prediction by miRanda. Expression profiles are derived from a comprehensive sequencing project of a large set of mammalian tissues and cell lines of normal and disease origin. In this prediction tool users can explore the set of genes that are potentially regulated by a particular microRNA.

PicTar: PicTar is a computational method for identifying common targets of microRNAs. It computes a maximum likelihood score that a given RNA sequence (typically a 3' UTR) is targeted by a fixed set of microRNAs. It uses a group of orthologous 3'UTRs from multiple species and then check complementarity to the seed region of a miRNA [47]. Matched alignments are then filtered according to their thermodynamic stability. Predicted targets are then scored using Hidden Markov Model (HMM) maximum-likelihood fit approach. This tool is well known for its excellent success rate in predicting targets for single microRNAs and for combinations of microRNAs.

RNAhybrid: It can identify multiple potential binding sites of miRNAs in target RNA sequence. RNAhybrid avoids intramolecular hybridizations, that is base pairings between target mRNA sequence or between miRNA sequence. Identified miRNA targets is assessed with an extreme value statistics of length normalized minimum free energies, a Poisson approximation of multiple binding sites, and the calculation of effective numbers of orthologous targets in comparative studies of multiple organisms [48].

\section{Conclusion}

miRNA acts as micro-regulator in biological system which plays an important role in modulating virtually all the biological processes. Identification of novel miRNAs and their targets may lead to better understanding of many regulatory processes inside the living systems. This is an emerging science with high futuristic impact. Computationally, target prediction is an attractive tool in studying the target genes which will be biologically validated later on. These bioinformatics algorithms help the researchers to identify the target genes and further study in relation with their biological function.

In conclusion, there is need to improve the accuracy of computational or bioinformatics tools for target gene identification, reduction of false positive targets and inclusion of experimentally validated targets.

\section{References}

1. Zhang, Z., Qin, Y. W., Brewer, G. and Jing, Q. (2012) MicroRNA degradation and turnover: regulating the regulators. WIRES RNA 3:593-600. 
2. Pritchard, C. C., Cheng, H. H. and Tewari, M. (2012) MicroRNA profiling: approaches and considerations. Nature Reviews Genetics 13, 358-369.

3. Pillai, R. S., Bhattacharyya, S . N. and Filipowicz, W. (2007) Repression of protein synthesis by miRNAs: how many mechanisms $\Delta$ Trends Cell Biol., 17, 118-126.

4. Ambros, V. (2004) The functions of animal microRNAs. Nature 431:350-355.

5. Rottiers, V. and Näär, A. M. (2012) MicroRNAs in metabolism and metabolic disorders. Nat Rev Mol Cell Biol. 13(4):239-50.

6. Bushati, N. and Cohen, S. M. (2007) microRNA functions. Annu. Rev.Cell Dev. Biol. 23, 175-205.

7. Lin, S. L., Miller, J. D. and Ying, S. Y. (2006) Intronic MicroRNA(miRNA). J Biomed Biotechnol. 26818.

8. Xiao, C. and Rajewsky, K. (2009) MicroRNA control in the immune system: basic principles. Cell 136:26-36.

9. Berezikov, E. (2011) Evolution of microRNA diversity and regulation in animals. Nat Rev Genet. 12(12):846-60.

10. Bartel, D. P. (2004) MicroRNAs: Genomics, Biogenesis, Mechanism, and Function. Cell 116 (2): 281 - 297.

11. Lee, R. C., Feinbaum, R. L., \& Ambros, V. (1993) The C. elegans heterochronic gene lin-4 encodes small RNAs with antisense complementarity to lin-14. Cell 75 (5):843 - 854.

12. Burnside, J. and Morgan, R. (2011) Emerging roles of chicken and viral microRNAs in avian disease. BMC Proc. 5 Supp14:S2.

13. Pfeffer, S., Sewer, A., Lagos-Quintana, M., Sheridan, R., Sander, C., Grässer, F. A., van Dyk, L. F., Ho, C. K., Shuman, S., Chien, M., Russo, J. J., Ju, J., Randall, G., Lindenbach, B. D., Rice, C. M., Simon, V., Ho, D. D., Zavolan, M., Tuschl, T. (2005) Identification of microRNAs of the herpesvirus family. Nat. Methods 2(4):269-76.

14. O'Connell, R. M., Rao, D. S., and Baltimore, D. (2012) microRNA regulation of inflammatory responses. Annu Rev Immunol. 30:295-312.

15. Friedman, R. C., Farh, K.K., Burge, C. B., Bartel, D. P. (2009) Most mammalian mRNAs are conserved targets of microRNAs. Genome Research; 19:92-105.

16. Miranda, K. C., Huynh, T., Tay, Y., Ang, Y. S., Tam, W. L., Thomson, A. M., Lim, B., Rigoutsos, I. (2006) A patternbased method for the identification of MicroRNA binding sites and their corresponding heteroduplexes. Cell 126: 1203-1217.

17. Huntzinger, E. and Izaurralde, E. (2011) Gene silencing by microRNAs: contributions of translational repression and mRNA decay. Nat Rev Genet. (2):99-110.

18. Lee, Y., Jeon, K., Lee, J. T., Kim, S., and Kim, V. N. (2002) MicroRNA maturation: stepwise processing and subcellular localization. EMBO 21 (17):4663 - 4670.

19. Nam, J. W., Shin, K. R., Han, J., Lee, Y., Kim, V. N., Zhang, B. T. (2005) Human microRNA prediction through a probabilistic co-learning model of sequence and structure. Nucleic Acids Res. 33(11) 3570-3581.

20. McDaneld, T. G. (2009) MicroRNA: mechanism of gene regulation and application to livestock. Journal of animal science 87:E21-E28.

21. Jackson, R. J., and Standart, N. (2007) How do microRNAs regulate gene expression $\triangle$ Sci. STKE 367: re1.

22. Buchan, J. R., and Parker, R. (2007) The two faces of miRNA. Science 318:1877-1878.

23. Shyu, A. B., Wilkinson, M. F., Hoof, A.V. (2008). Messenger RNA regulation: To translate or to degrade. EMBO J. 27:471-481.

24. Wu, L., Fan, J., Belasco, J.G. (2006) MicroRNA direct rapid deadenylation of mRNA. Proc. Natl. Acad. Sci. USA 103: 4034-4039.

25. Thermann, R., and Hentze, M. W. (2007) Drosophila miR2 induces pseudo-polysomes and inhibits translation initiation. Nature 447:875-878.

26. Kiriakidou, M., Tan, G. S., Lamprinaki, S., Planell-Saguer, M. D., Nelson, P. T., Mourelatos, W. (2007) An mRNA m7G cap binding-like motif within human Ago2 represses translation. Cell 129:1141-1151.

27. Chan, S. P., and Slack, F. J. (2006) microRNA-mediated silencing inside P-bodies. RNA Biol. 3:97-100.

28. Griffiths-Jones, S., Grocock, R. J., Dongen, S.V., Bateman, A. and Enright, A. J. (2005) miRBase: microRNA sequences, targets and gene nomenclature. Nucleic Acids Research 34 (suppl 1): D140-D144.

29. Yoon, S., and Micheli, G. D. (2006) Computational identification of microRNAs and their targets. Birth Defects Research Part C: Embryo Today: Reviews 78 (2):118-128.

30. Maziere, P. and Enright, A. J. (2007) Prediction of microRNA targets. Drug Discovery Today 12 (11):452 - 458.

31. Bartel, D. P. (2009) MicroRNAs: target recognition and regulatory functions. Cell. 136:215-233.

32. Xiao, F., Zuo, Z., Cai, G., Kang, S., Gao, X., \& Li, T. (2009) miRecords: an integrated resource for microRNA-target interactions. Nucleic Acids Research 37:D105-D110.

33. Kertesz, M., Iovino, N., Unnerstall, U., Gaul, U. and Segal, E. (2007) The role of site accessibility in microRNA target recognition. Nat Genet. 39: 1278-1284.

34. Hofacker, I. L. (2007) How microRNAs choose their targets. Nat Genet. 39: 1191-1192.

35. Lewis, B. P., Burge, C. B. and Bartel, D. P. (2005) Conserved seed pairing, often flanked by adenosines, indicates that thousands of human genes are microRNA targets. Cell; 120(1): 15-20.

36. Grimson, A., Farh, K.K., Johnston, W.K., Garrett-Engele, P., Lim, L.P., and Bartel, D.P. (2007) MicroRNA targeting specificity in mammals: determinants beyond seed pairing. Mol. Cell 27:91-105.

37. Lim, L.P., Lau, N.C., Weinstein, E.G., Abdelhakim, A., Yekta, S., Rhoades, M.W., Burge, C.B. and Bartel, D.P. (2003) The microRNAs of Caenorhabditis elegans. Genes \& Dev., 17: 991-1008.

38. Bernhart, S. H., Tafer, H., Mückstein, U., Flamm, C., Stadler, P. F and Hofacker, I. L. (2006) Partition function and base pairing probabilities of RNA heterodimers. Algorithms $\mathrm{Mol}$ Biol. 1(1):3.

39. Lewis, B. P., Shih, I., Jones-Rhoades, M. W., Bartel, D. P. and Burge, C. B. (2003) Prediction of Mammalian MicroRNA Targets. Cell, 115(7), 787-798.

40. Enright, A. J., John, B., Gaul, U., Tuschl, T., Sander, C. and Marks, D. S. (2004) MicroRNA targets in Drosophila. Genome Biol. 5(1), 1.

41. Wang, X. (2008) miRDB: a microRNA target prediction and functional annotation database with a wiki interface. $R N A 14$ (6), 1012-1017.

42. Krek, A., GrÄun, D., Poy, M. N., Wolf, R., Rosenberg, L., Epstein, E. J., MacMenamin, P., da Piedade, I., Gunsalus, K. C. and Stoffel, M. (2005) Combinatorial microRNA target predictions. Nat. Genet. 37, 495-500.

43. Kim, S. K., Nam, J. W., Rhee, J. K., Lee, W. J. and Zhang, B. T. (2006) miTar- get: microRNA target gene prediction using a support vector ma- chine. BMC Bioinform., 7(1), 411.

44. Yue, D., Liu, H. and Huang, Y. (2009) Survey of Computational Algorithms for MicroRNA Target Prediction. Current Genomics, 10, 478-492.

45. Long, J. M. and Lahiri, D. K. (2012) Advances in microrna experimental approaches to study physiological regulation of gene products implicated in cns disorders. Exp. Neurol., 235: 402-418.

46. Selbach, M., Schwanhausser, B., Thierfelder, N., Fang, Z., Khanin, R. and Rajewsky, N. (2008) Widespread changes in protein synthesis induced by microRNAs. Nature. 455: 58-63.

47. Krek, A., Grun, D., Poy, M. N., Wolf, R., Rosenberg, L., Epstein, E. J., Macmenamin, P., da Piedade, I., Gunsalus, K. C., Stoffel, M. and Rajewsky, N. (2005) Combinatorial microRNA target predictions. Nat Genet. 37: 495-500.

48. Rehmsmeier, M., Steffen, P., Chsmann, M. H. and Giegerich, R. (2004) Fast and effective prediction of microRNA/target duplexes. RNA 10:1507-1517.

$* * * * * * * *$ 